\section{A Patient with a Phenotype of Adult-onset Still Disease, But a Genotype Typical of Cryopyrin-associated Periodic Fever Syndrome}

\section{To the Editor:}

Cryopyrin-associated periodic fever syndrome (CAPS) is a group of genetic diseases consisting of familial cold autoinflammatory syndrome (FCAS) and presenting with urticaria triggered by cold, Muckle-Wells syndrome (MWS) with fever, hearing loss, rash and joint pain, and neonatal onset multisystem inflammatory disease (NOMID), a severe neonatal disease. CAPS is associated with mutations in the NLRP3 gene ${ }^{1,2}$. NLRP3 associates with the proteins ASC and pro-caspase 1 to form the NLRP3 inflammasome, which is important for activation of pro-interleukin $1 \beta$ (IL-1 $\beta$ ) to mature IL-1 $\beta$. Consequently, CAPS can be treated with anti-IL-1 $\beta$ therapy. Some patients with more diffuse inflammatory symptoms together with NLRP3 mutations have been classified as "atypical" CAPS ${ }^{3}$. We describe a patient with inflammatory symptoms fulfilling criteria of adult-onset Still disease (AOSD) but with a genotype typical of CAPS

A 32-year-old man with previously known thrombophlebitis related to activated protein $\mathrm{C}$ resistance presented in May 2009 with sore throat, relapsing fever above $39^{\circ} \mathrm{C}$ especially in the afternoon, cervical adenitis, and transient maculopapular exanthema. C-reactive protein (CRP) was 192 $\mathrm{mg} / \mathrm{l}$, leukocyte count $11.3 \times 10^{9} / 1$ (ref < 8.8), neutrophils $8.2 \times 10^{9} / 1$, aspartate aminotransferase $1.6 \mu \mathrm{kat} / 1$ (ref <0.76), alanine aminotransferase $2.5 \mu \mathrm{kat} / 1$ (ref $<1.2)$, and lactate dehydrogenase $9.7 \mu \mathrm{kat} / 1(\mathrm{ref}<3.5) . \mathrm{IgG}$ antibodies against Epstein-Barr virus (EBV) were present, but EBV, cytomegalovirus, and other infections could not be detected. Three weeks after presentation, CRP was $54 \mathrm{mg} / \mathrm{l}$; the patient felt better, but relapsing exanthema, tiredness, and low-grade fever continued.

In September 2010 , sore throat, fever above $39^{\circ} \mathrm{C}$, cervical adenitis, and exanthema relapsed, and in addition the patient presented with arthralgia, gonarthritis, and tenosynovitis of the right palmar hand. A faint red maculopapular rash appeared transiently, but it was neither urticaria-like nor typically salmon pink (Figure 1). No symptoms were induced by cold, whereas physical activity or psychological stress seemed to trigger flares. CRP was $214 \mathrm{mg} / \mathrm{l}$, serum ferritin $5118 \mu \mathrm{g} / \mathrm{l}$ (ref 34-275), and leukocyte count $13.6 \times 10^{9} / 1$ (neutrophils $10.6 \times 10^{9} / \mathrm{dl}$ ). Liver enzymes were normal at this occasion. Rheumatoid factor and antinuclear antibodies were negative. Serum amyloid A levels were within the normal range at 2 occasions. Urine albumin was never detected on dipstick examinations. Computed tomography examinations of thorax and abdomen were normal, and malignancy or infections could not be detected. AOSD was

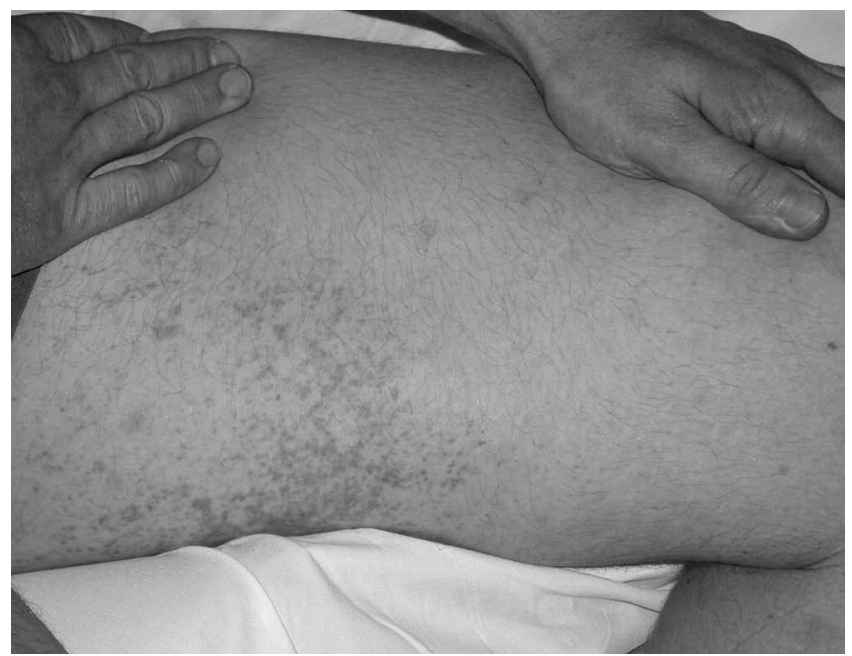

Figure 1. Transient maculopapular exanthema appeared during disease relapse in January 2012. diagnosed according to the classification criteria of Yamaguchi, et $a l^{4}$ Table 1 shows different findings compatible with AOSD and/or CAPS. The clinical picture was not specific for FCAS, MWS, or NOMID, and the family history was negative. Audiogram was normal in 2012. No previous episodes of hearing loss or eye symptoms were reported.

The patient was given prednisolone $40 \mathrm{mg}$ daily, with no relief of symptoms. Subcutaneous injections of anakinra $100 \mathrm{mg} /$ day were started and 6 days later the fever ceased, CRP was $<10 \mathrm{mg} / \mathrm{dl}$, and serum ferritin decreased to $1519 \mu \mathrm{g} / \mathrm{dl}$. However, anakinra injections caused severe local skin reactions with fever, increasing levels of CRP (80 mg/dl), and serum ferritin $(4818 \mu \mathrm{g} / \mathrm{dl})$. Treatment with anakinra was stopped in October 2010 and trials to reintroduce anakinra were unsuccessful. With single therapy of prednisolone $40 \mathrm{mg} /$ day, arthralgia and exanthema continued intermittently, although CRP normalized and serum ferritin decreased to $800 \mu \mathrm{g} / 1$

From November 2010 to April 2011 etanercept was used, and prednisolone was tapered from $30 \mathrm{mg}$ to $5 \mathrm{mg} /$ day. CRP and ferritin levels were in the normal range, but symptoms from joints and skin continued. In March 2011 the symptoms deteriorated, with sore throat, migrating arthralgia, and exanthema, and serum ferritin rose to $1211 \mu \mathrm{g} / \mathrm{l}$ Prednisolone was increased to $40 \mathrm{mg} / \mathrm{day}$, and in April 2011 intravenous tocilizumab $800 \mathrm{mg}$ every second or third week was introduced. The infusions were complicated by thrombophlebitis and the clinical effect was only partial. In December 2011, the original symptoms relapsed in spite of increased prednisolone dose from minimum 10 to $30 \mathrm{mg} / \mathrm{day}$, and eventually tocilizumab was discontinued.

In January 2012, subcutaneous canakinumab (an antibody against

Table 1. Symptoms and signs in the patient indicative of adult-onset Still's disease (AOSD) and CAPS. Classification criteria for AOSD according to Yamaguchi require 5 or more criteria, with at least 2 being major (item 1-4; item 5-8 are minor criteria). Exclusion criteria are infections (especially sepsis and infectious mononucleosis), malignancy (especially malignant lymphoma), and rheumatic diseases (especially polyarteritis nodosa and rheumatoid vasculitis with extraarticular features). All criteria are applicable only in the absence of other clinical explanations. The rash in AOSD is often salmon-pink and usually appears during fever.

\begin{tabular}{llcc}
\hline Feature & AOSD & CAPS \\
\hline 1 & Fever of $39^{\circ}$ C or higher, lasting 1 week or longer & Yes & Yes \\
2 & Arthralgia lasting 2 weeks or longer & Yes & Yes \\
3 & Macular or maculopapular nonpruritic rash & Yes & Yes \\
4 & Leukocytosis $\left(10 \times 10^{9} / 1\right.$ or greater) with & & \\
$\quad \geq 80 \%$ granulocytes & Yes & Yes \\
5 & Sore throat & Yes & - \\
6 & Lymphadenopathy and/or splenomegaly & Yes & - \\
7 & Abnormal liver function tests with elevated & & \\
$\quad$ levels of transaminases and/or lactate & & \\
$\quad$ dehydrogenase not attributed to drug & & Yes \\
$\quad$ allergy/toxicity or other causes & Yes & Yes \\
8 & Negative ANA and IgM RF & Yes & No \\
9 & High ferritin levels & Yes & Yes \\
10 & Response to IL-1 inhibition & & \\
11 & Response to moderate to high doses of & Yes & - \\
12 & Family history & - & No \\
13 & Urticaria & - & No \\
14 & Cold-induced skin symptoms & No \\
16 & Conjunctivitis or other eye symptoms & No \\
\hline
\end{tabular}

CAPS: cryopyrin-associated periodic fever syndrome; ANA: antinuclear antibodies; RF: rheumatoid factor; IL: interleukin. 
IL-1 $\beta) 150 \mathrm{mg}$ every eighth week was started. The patient immediately felt better and all joint and skin symptoms disappeared. CRP, ferritin, and leukocyte levels returned to normal and have been normal since then. Prednisolone was stopped in July 2012 and he was able to resume long-distance running.

Gene sequencing of exon 3 of the NLRP3 gene revealed a heterozygous mutation (c.778C > T) in position R260W, typically found in CAPS ${ }^{2}$. He was also heterozygous for c.2107C >A (p. Q703K, rs35829419), a known gene polymorphism with an allele frequency of $6.5 \%$ in a Swedish population $^{5}$. The importance of the Q703K polymorphism is difficult to judge because it has been reported in healthy individuals and patients with fever ${ }^{5}$. However, functional tests have revealed high IL-1 $\beta$ production from THP-1 cells with the R260W mutation, and moderately increased levels for the Q703K polymorphism compared to wild-type ${ }^{6}$.

The patient did not tolerate the IL-1 receptor antagonist anakinra, whereas the effect of the anti-IL-1 antibody canakinumab was remarkable, supporting the pathogenic importance of IL- $1 \beta$ in this case. Anti-IL-1 therapy is useful in CAPS but also in $\operatorname{AOSD}^{7}$, although patients with AOSD usually do not present mutations in the NLRP3 gene ${ }^{8,9}$. The symptoms of AOSD and CAPS partly overlap (Table 1), so different sets of diagnostic criteria may be challenging to use. Our patient fulfilled the Yamaguchi criteria, responded partially to higher doses of steroids, and had high ferritin levels, supporting phenotypic AOSD. The rash was indicative of AOSD, but rash as well as fever and response to IL-1 inhibition may also be seen in CAPS. Many patients with CAPS do not have a family history of CAPS or have symptoms triggered by cold. To our knowledge this is the first report of a patient with a phenotype compatible with AOSD but a genotype typical of CAPS

PER ERIKSSON, MD, Associate Professor; CLAUDIA JACOBS, MD, Division of Rheumatology; PETER SÖDERKVIST, PhD, Professor, Division of Cell Biology, Department of Clinical and Experimental Medicine, Faculty of Health Sciences, Linköping University, Linköping, Sweden. Address correspondence to Dr. P. Eriksson, Department of Rheumatology, University Hospital, 58185 Linköping, Sweden.

E-mail: per.eriksson@lio.se

\section{REFERENCES}

1. Hoffman HM, Mueller JL, Broide DH, Wanderer AA, Kolodner RD. Mutation of a new gene encoding a putative pyrin-like protein causes familial cold autoinflammatory syndrome and Muckle-Wells syndrome. Nat Genet 2001;29:301-5.

2. Dodé C, Le Dû N, Cuisset L, Letourneur F, Berthelot JM, Vaudour $\mathrm{G}$, et al. New mutations of CIAS1 that are responsible for Muckle-Wells syndrome and familial cold urticaria: A novel mutation underlies both syndromes. Am J Hum Genet 2002;70:1498-506.

3. Verma D, Eriksson P, Sahdo B, Persson A, Ejdebäck M, Särndahl E, et al. Two adult siblings with atypical cryopyrin-associated periodic syndrome due to a novel M299V mutation in NLRP3. Arthritis Rheum 2010;62:2138-43.

4. Yamaguchi M, Ohta A, Tsunematsu T, Kasukawa R, Mizushima Y, Kashiwagi H, et al. Preliminary criteria for classification of adult Still's disease. J Rheumatol 1992;19:424-30.

5. Verma D, Lerm M, Blomgran Julinder R, Eriksson P, Söderkvist $P$, Särndahl E. Gene polymorphisms in the NALP3 inflammasome are associated with interleukin-1 production and severe inflammation: Relation to common inflammatory diseases? Arthritis Rheum 2008;58:888-94.

6. Verma D, Särndahl E, Andersson H, Eriksson P, Fredrikson M, Jönsson J-I, et al. The Q705K polymorphism in NLRP3 is a gain-of-function alteration leading to excessive interleukin-1 $\beta$ and IL-18 production. PLoS One 2012;7:324977.

7. Nordström D, Knight A, Luukkainen R, van Vollenhoven R, Rantalaiho V, Kajalainen A, et al. Beneficial effect of interleukin 1 inhibition with anakinra in adult-onset Still's disease. An open, randomized, multicenter study. J Rheumatol 2012;39:2008-11.

8. Goldbach-Mansky R. Immunology in clinic review series; focus on autoinflammatory diseases: update on monogenic autoinflammatory diseases: The role of interleukin (IL)-1 and an emerging role for cytokines beyond IL-1. Clin Exp Immunol 2012;167:391-404.

9. Hayem F, Hayem G. Still's disease and the mitochondrion: The other face of an old friend? Med Hypotheses 2012;79:136-7.

J Rheumatol 2013;40:9; doi:10.3899/jrheum.130325 